\title{
Design and Research of Real Time Monitoring System for the Refined Oil Logistics
}

\author{
Juntao Li ${ }^{1}$, Xianzhong $Q i^{2}$ \\ ${ }^{1}$ Information Institute, Beijing Materials Institute, Beijing, China \\ ${ }^{2}$ Department of Logistics Engineering, Beijing Materials Institute, Beijing, China
}

Email address:

qixianzhong@Chinabochen.com (Xianzhong Qi)

\section{To cite this article:}

Juntao Li, Xianzhong Qi. Design and Research of Real Time Monitoring System for the Refined Oil Logistics. Journal of Investment and Management. Vol. 6, No. 1, 2017, pp. 1-5. doi: 10.11648/j.jim.20170601.11

Received: October 4, 2016; Accepted: October 20, 2016; Published: November 14, 2016

\begin{abstract}
Dangerous goods transport is very dangerous. Once the dangerous goods accidents will be on human life and the environment cause significant harm and loss. Therefore, the safe transport of dangerous goods is very important. In my paper, the logistics monitoring system is designed for the logistics process of the refined oil. At First, the paper analyzes the present situation of dangerous goods logistics and logistics monitoring technology both at home and abroad. Secondly, we investigated the logistics monitoring technology, such as GPS/RFID/GPRS and so on. Next, we studied the characteristics of the refined oil, the mode of logistics and the business process of the recycling logistics. Finally, basing on the Internet of things, the software and hardware of the monitoring system is designed to ultimately achieve the purpose of the real-time monitoring in the refined oil logistics process, which improves the safety and the efficiency of information transmission in the process of transportation. The paper mainly studies the software and hardware configuration of the monitoring system. The next paper will be on the monitoring system software design for further study.
\end{abstract}

Keywords: Dangerous Goods Logistics, Logistics Monitoring System, The Real-Time Monitoring, The Refined Oil Logistic, The Internet of Things

\section{Introduction}

Dangerous goods, namely chemicals abbreviation, refers to flammable, explosive, corrosive and other hazardous properties of chemicals [1]. At present, Dangerous Goods Logistics is still in its infancy in China, and most of the modes of logistics operation are still in the traditional logistics modes. Because of the backwardness of the dangerous goods logistics monitoring technology, accidents occur from time to time, the danger caused a significant threat for people and the environment [2]. In recent years, the accidents of dangerous goods frequently occur in China, causing great damage on the surrounding population, flora, fauna and the environment [3].

There are many factors that caused the dangerous goods accidents, such as inadequate governmental monitoring and low quality of employees, inadequate monitoring technology and post-processing not being timely and so on [4]. In view of this situation, both the executive branch and the logistics business have to make the appropriate policies and measures to reduce accidents and improve the safety of dangerous goods logistics.

In recent years, foreign vehicle monitoring has done a very extensive study. On the basis of these studies, many countries have developed a number of specialized commodities. Such as the Japanese tire pressure sensor and tilt sensor. These sensors integrate a variety of sensor data, while the sensor can be integrated to determine the process of driving the vehicle potential hazards and hidden faults. North American company developed the tire electronic detection devices. Induction piece is installed into the tire, the tires can work tire pressure and temperature data. In the process of transporting dangerous goods, most foreign companies are using GPS technology to monitor the vehicle driving routes. The sensor data is then transmitted to the control center via the network. Finally, we can achieve the purpose of real-time monitoring of vehicles. 


\section{System Functions Brief Description and the Overall Structure}

In view of the danger and problems in the process of logistics of dangerous goods and the basic requirement of building a unified information monitoring platform, logistics monitoring system in this paper should meet the two key indexes,namely improving the efficiency of logistics and safety of oil products. In the implementation process, basing on the Internet of things, we make use of the technologies of Internet of things, mainly setting the systems from two aspects of software systems and hardware systems.

(1) Hardware system

Dangerous goods will be loaded with different RFID label, the vehicle sensor inside can sense the speed, temperature, humidity, smoke and angle of dangerous goods [5]. In case of the danger, the vehicle sensor will control self- regulating or feedback to the driver and the control center. Each vehicle is equipped with GPS the selection optimal path, monitoring the status and position of the vehicle at any time in order to adjust the traffic route to ensure the timely delivery of dangerous goods to the destination. Each vehicle is equipped with a GPRS terminal, which is mainly connected with the GPRS network, and the collected information is sent to the monitoring center. Dangerous goods warehouse storage system consists of video security module, environment control module, access control module, material terminal module and patrol module, implementing the environmental monitoring, improving the safety level of warehouse, preventing security risks caused by dangerous goods and the goods losing risk.

(2) Software system

The software of monitoring system is mainly made up of the monitoring terminal software module and the monitoring center software module. Through the configuration of multisystem software, we can control the monitoring terminal, make the rules and control monitoring data of filtration, storing and analysis, and so on, and the software can help manage the staff.

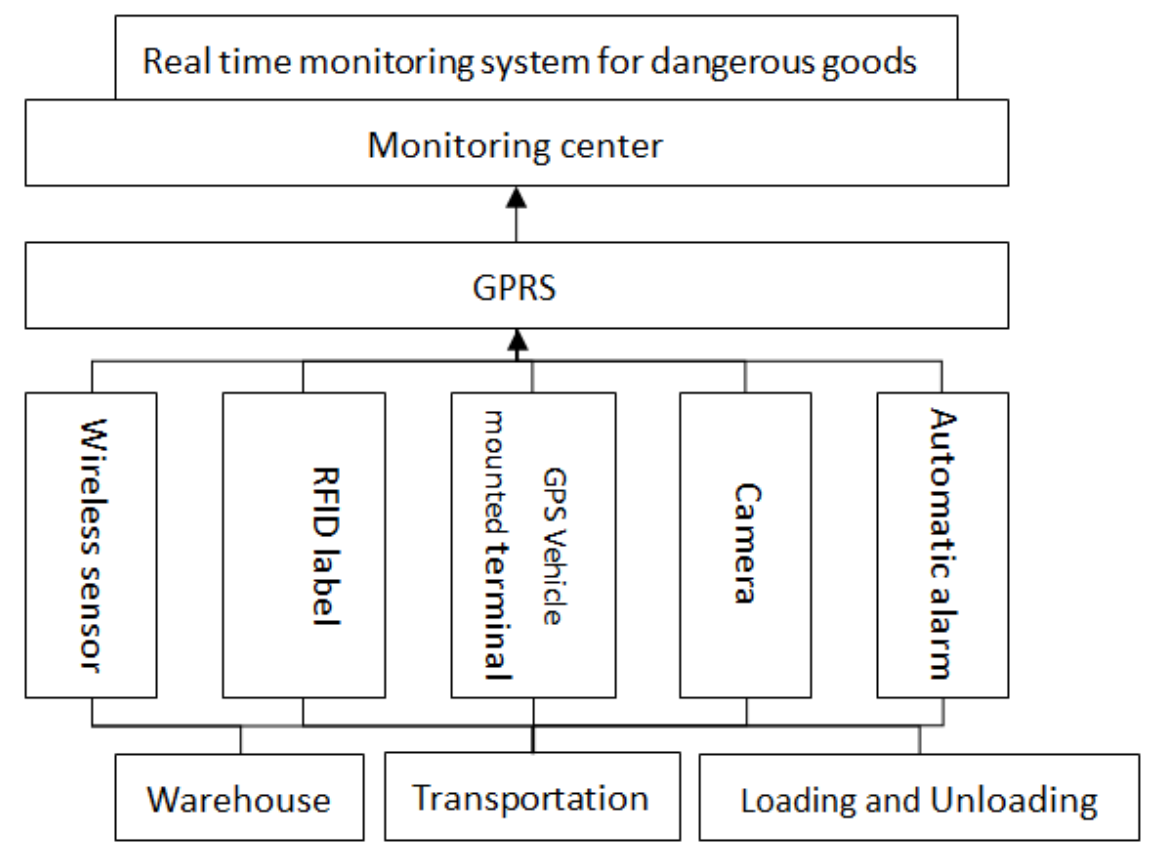

Fig. 1. The system overall framework.

\section{System Hardware Configuration}

\subsection{Configuration Principle}

Based on the actual situation, the design takes full account of the current specific situation of the refined oil transportation, and makes the specific monitoring plan to improve the safety of the finished oil logistics. In the tank car, using wireless sensor devices, it has the advantages of real-time monitoring that timely transferring information of RFID technology and GPS technology to the monitoring center through the GPRS technology. The monitoring center can timely monitor the refined oil warehouse status and know the status of the logistics in the transport process of refined oil, for guaranteeing the safety of the goods. Even if the accident occurred, the monitoring center can monitor information to conduct on-site rescue, reducing the accident loss.

\subsection{Program Content}

According to the design principles, the logistics- process of finished oil and risk analysis, and considering the actual situation and the future development trend of the project, we construct configuration scheme monitoring process for this project.

In the logistics-process of finished oil, the logistics link is more complex with great risk of the logistics-process, so the monitoring system set in the logistics of warehousing, transportation, loading and unloading and other links. Specific monitoring hardware system diagram are shown in figure 2 : 


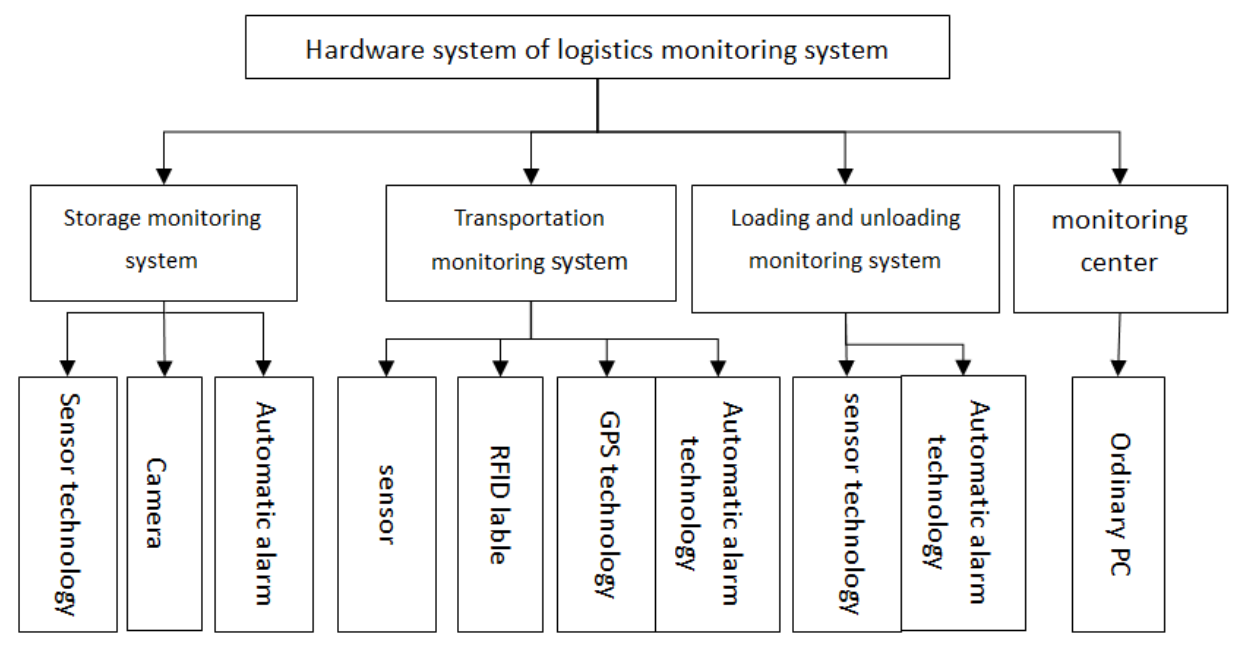

Fig. 2. The hardware design scheme of logistics monitoring system.

\subsection{Configuration Description}

(1) The sensor module

The wireless sensor is installed outside of the oil tank. when loading and unloading the refined oil, through the sensor measuring oil flow timely, the real-time refined oil liquid level information is transmitted to the site through the wireless sensor network in order to realize the automatic monitoring of loading and unloading the refined oil, avoiding taking tank accidents and eliminating overload. After the completion of the loading liquid into vehicle, we write the degree of risk, emergency treatment and oil products of unit information, the receiving unit Information together into the electronic tag on the vehicle for future reference.

In the storage-process by analysis technology of vibration sensing, the sensor is installed outside of the oil tank. we use of the oil pressure sensor measuring the refined oil liquid level in the oil tank, temperature, pressure and real-time monitoring refined oil products in the process of oil storage conditions. It can also be determined by the refined oil liquid level of the tank to determine whether the leakage of liquid.

(2) RFID technology module

In the logistics-process of finished oil, the transport vehicles are labeled with different electronic tags (i.e. different ID), which are perceived the condition of the dangerous goods by the vehicle sensor. In transport, RFID reader is set up along the expressway and the toll station, which can monitor the vehicle [6]. In transit, data is returned to the control center at any time through the wireless network. If abnormal situation occurs, this technology can immediately carry on the simple self-adjustment to the car cabin or feed back to the personnel and vehicle control center in order to make corresponding modification and the coordination. This system cam start the alarm if the abnormality is serious, and feed adjustment the whole process state information. Monitoring center uses the database to conduct data collecting, showing the effective information, so that the staff make judgement timely to ensure safe and successful operation [7].
(3) GPS technology module

This system uses the M GPS a type 89 HOLUX receiving module. The demand of refined oil transport vehicles for GPS system is shown to ensure the safety of the transport process, dealing with alarm information quickly, controling the vehicle running route, area and speed, etc. Through the integration with GPS and short message system, the whole process management of finished oil in transit or storage condition is realized. In the process of transportation, GPS terminal installed in the vehicles monitors the position and speed of the vehicle through the GPS system, alarming when the vehicle is off regulation route or speeding. All of these vehicles travelling information will be transmitted through the built-in GPRS mobile communication system to remote monitoring stations, so as to achieve the national remote tracking and monitoring of the vehicle.

(4) Automatic alarm technology module

For the safety information monitoring of finished oil, automatic alarm positioning management module is inevitable to use the related detection and alarming equipment. For example, the liquid leakage detection. Therefore, in the development of the system, we should fully consider the applied management, data acquisition and information integration of automatic control equipment, to provide more in-depth and comprehensive monitoring measures to improve the security of the logistics-process.

(5) Video monitoring technology module

Considering the control of image acquisition module, a model for the GXT.M201 serial camera is a video image acquisition module and image compression function, with 300 thousand pixels CMOS camera, maximum resolution of $640 * 480$, containing image acquisition device control, video capture, image acquisition, image data compression JPEG serial communication, such as full-featured shooting.

For high risk, major chemicals warehouse, the video monitoring technology had better take 24 hours of video surveillance point. Under normal circumstances, the storage management is very strict for environmental requirements, 
such as the special requirements of temperature and humidity and display etc.. Under normal circumstances, the video monitoring module is installed in the finished oil warehouse. Video surveillance system is composed of the camera and video surveillance equipment, with video surveillance, video storage, video playback and other functions. Application of video monitoring system improved the monitoring ability of the warehouse, real-time video monitoring of dangerous goods, to prevent the illegal outflow of dangerous goods, and greatly improved the security level of the warehouse.

(6) GPRS technology module

System uses the MC22 as the remote transmission of GPRS module. GPRS is the chain part of the monitoring system, which links the vehicle and warehouse monitoring system and the remote control center of the state information exchange. The information is sent out by the vehicle GPRS terminal and remote monitoring center through the GPRS receiver receives and import real-time information into the database through the information transmission module and data interface. Database records real-time data information and summarizes information. Then, expert decision-making system put as background decision support, the conclusion is obtained finally [8].

(7) Hardware description of the monitoring center

The monitoring center system uses the common PC server as the center server, and its performance parameters are shown in Table 1:

Table 1. The table of Server performance parameters

\begin{tabular}{ll}
\hline CPU Type & Intel Corp Pentium4 \\
\hline CPU Frequency $(\mathrm{MHz})$ & $2.4 \mathrm{~GB}$ \\
CPU Second Level Cache & $212 \mathrm{~KB}$ \\
Memory type & $\mathrm{DDR} 2$ \\
Memory size & $1 \mathrm{~GB}$ \\
Hard disk size & $120 \mathrm{~GB}$ \\
Processor FSB & $133 \mathrm{MHz}$ \\
\hline
\end{tabular}

\section{System Software Configuration}

\subsection{Configuration Principle}

The design combined with the actual situation of refined oil logistics, making full use of the existing system, makes the specific software design and builds an efficient software system, for the sake of fast transmission and process information, controlling monitoring system, reducing reinvestment of the enterprise in the information system, meeting easy integration, easy updating and expansion principle. Efficient software system completes the processing of information and the control of the logistics process and improves the efficiency and safety of refined oil logistics.

\subsection{Program Content}

The logistics of finished oil monitoring system will use web service technology to realize the service oriented architecture (Oriented Architecture Service, abbreviated as SOA). SOA is a kind of system structure which is international and advanced information system. The software structure of the monitoring terminal of the system is shown in figure 3:

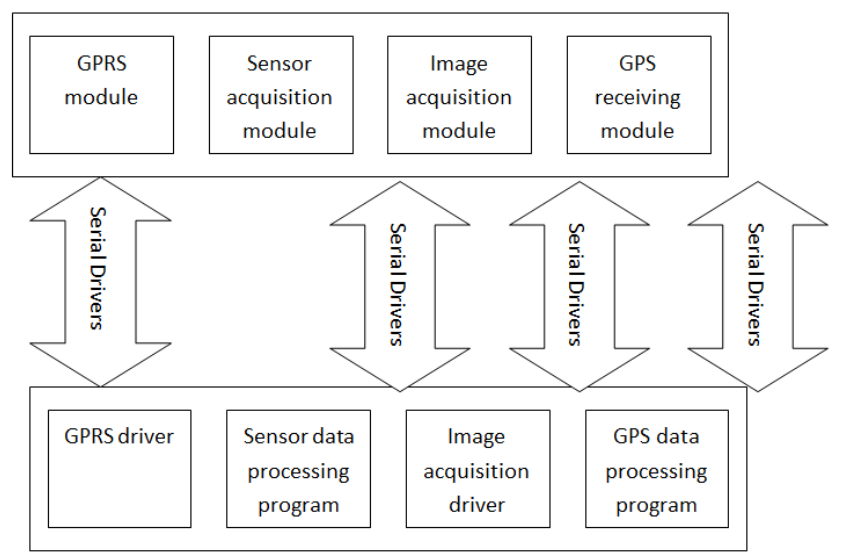

Fig. 3. Monitoring terminal software composition.

The software structure of the monitoring center of the system is shown in figure 4:

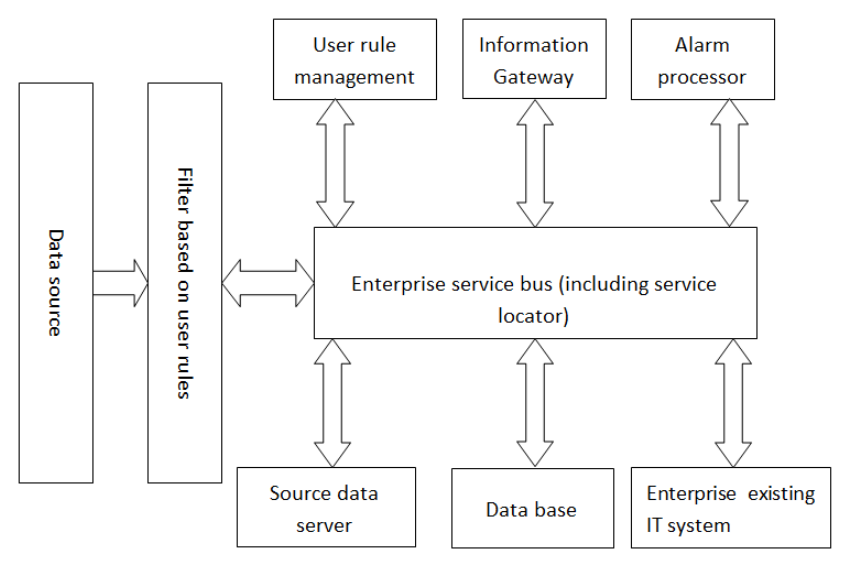

Fig. 4. The software design scheme of logistics monitoring system.

\subsection{Configuration Description}

(1) Monitoring terminal software description

Figure 2. is the structure of the software system of the monitoring system. This system uses the module type integration method to collect the data of the terminal. Each acquisition module corresponds to a group of driver, connecting different serial port with data acquisition module and the driving program, so that the data transmission between the two modules is very regular, easy operation, easy acquisition and easy correction. This software provides the interface of GPRS and Internet. After connecting the Internet network, the software can transfer the collected data into the monitoring center for processing.

(2) Software description of the monitoring center

1) Data source. This module can reliably obtain real raw data from the sensor, RFID system and GPS system. If the anti-jamming of the hardware in the terminal is poor or poor accuracy of reading, this module can also make a correct at the software level, to ensure the accuracy of the data source.

2) Filter based on user's rule. Because of the 
information obtained from the monitoring terminal being a large amount of unsettled data, which has a lot of redundant data, there is no sense of the logistics process. After receiving a large amount of information, the system can filter the information according to the specified principles of the user the actual situation, and provide the meaningful data for the system.

3) User rule manager. Because of the filter requiring filtering rules, users can use this manager to develop a variety of rules, such as the complex rules formulated by complex event processor. Abnormal event processor can make abnormal event rules. The rules of spatial database should formulate safe path design rules.

4) The alarm processor. This manager specializes in handling abnormal events, especially high risk events. For each of the abnormal events of the system record, there will be a predesigned treatment plan and the corresponding responsibility and decision makers. When an event occurs, the prepared program will be sent to the relevant staff of emergency event, and the relevant departments will be informed timely in the form of SMS or email notification.

5) The information portal. The function of this module is to collect all kinds of important information and extract the key knowledge from a large amount of information, through the digital dashboard and other technology revealed. Then, this module help the decision maker to have a comprehensive and clear understanding of the whole system in order to make the right decision [9].

6) The source data server. The server manages semantic information. Making full management of it will help the whole system to run.

7) The database. Database is the data storage part of dangerous goods transportation monitoring system, eventually easy access to database for data access. Database also has a good database security mechanism. The protection of database data is also very important, so it is necessary to establish a unified database security mechanism meanwhile there need to establish access to the system to prevent user access to other rights of the data. The establishment of the database can also ensure the reduction of redundant data storage. The data stored in the database can only be stored once. So the performance of the database directly affects the performance of the whole monitoring system.

8) The enterprise IT system. On the basis of analyzing the existing IT system of the existing enterprises, the function of the other valuable services is to be packaged into Web services. In addition, in personnel management function, this system can record the history of the training of all employees and the authorized [10] to deal with dangerous goods.

\section{Conclusions}

The real time monitoring system for the refined oil logistics is one of the visual logistics monitoring system. This paper mainly studies the design and implementation of the monitoring system of the refined oil logistics. Firstly, the structure of the monitoring system is designed. Secondly, the article describes the hardware configuration and software setting, and solves the problem of logistics monitoring of the refined oil in the secondary logistics process. The system clearly defines the process of oil product logistics monitoring. The system is designed to avoid accidents caused by poor information and improve the security of logistics.

\section{References}

[1] Yanju $\mathrm{Hu}$, Yanling $\mathrm{Hu}$, Wei Zhang. Study on emergency decision system of hazardous chemical accidents. Journal of Jilin Institute of Chemical Technology. 2010. 27(1): 69-72.

[2] Xin Zhao. Research on the construction of the monitoring platform for the transportation of dangerous goods [D]. Liaoning: Dalian Maritime University, 2013.

[3] Xin Yin. The gap analysis of the development of dangerous goods logistics at home and abroad [J]. Chongqing: Chongqing University, 2009.

[4] Genping Ma. Optimization of China's petroleum product logistics [J]. Shanghai: Shanghai Jiaotong University, 2008.

[5] Bin Xu. On the transportation of refined oil in the process of building safety management mechanism [J]. Beijing: Tsinghua University, 2009.

[6] Yadong Wang. Logistics security monitoring system based on RFID technology [J]. Gansu: Lanzhou University, 2010.

[7] Yadong Wang. Design and Realization of Chemical Dangerous Goods Logistics Safety Monitoring System Based on RFID Technology [D]. Lanzhou: Lanzhou University, 2010.

[8] Yadong Wang. Logistics safety monitoring system based on RFID technology [J]. Gansu: Lanzhou University, 2010.

[9] James Huddleston. Beginning C\#2002 Dambases Frcan Novice to Professional [M]. Beijing: Publishing House of Tsinghua University, 2008.

[10] Based on the universal network bears traces of waterway dangerous goods transportation monitoring system research and design [J]. Wuhan: Wuhan University of Technology 2010. 